\title{
A comparative study of functional outcome between posterior lumbar fusion and posterior lumbar interbody fusion in spondylolisthesis
}

\author{
Harpreet Singh, Dhruv Patel*, Sangam Tyagi, \\ Krushna Saoji, Tilak Patel, Parth Patel
}

Department of Orthopaedics, Geetanjali Medical College and Hospital, Udaipur, Rajasthan, India

Received: 09 March 2021

Revised: 12 April 2021

Accepted: 13 April 2021

\section{*Correspondence:}

Dr. Dhruv Patel,

E-mail: dhruvpateld2408@gmail.com

Copyright: ( $)$ the author(s), publisher and licensee Medip Academy. This is an open-access article distributed under the terms of the Creative Commons Attribution Non-Commercial License, which permits unrestricted non-commercial use, distribution, and reproduction in any medium, provided the original work is properly cited.

\begin{abstract}
Background: Spondylolisthesis is condition in which one vertebra slips over other vertebra. This study has been done to compare the functional outcome and complications of two techniques: posterior lumbar fusion (intertransverse fusion) and posterior lumbar interbody fusion.

Methods: Total 20 patients with spondylolisthesis admitted in a tertiary care centre in Rajasthan were allotted alternatively in posterior lumbar fusion (PLF) group and posterior lumbar interbody fusion (PLIF) group. In PLF, fusion was done by placing bone graft between transverse processes and around facets. In PLIF, fusion was bone by placing cage in between vertebral bodies.

Results: 20 patients were included in our study with female predominance (65\%). Mean age was 54.2 years (PLF=58.4 and PLIF=50.2). 70\% patients have L4-L5 level spondylolisthesis. Average operative time was less in PLF group, which is statistically significant. Functional outcome was measured by using visual analogue scale (VAS) score and Japanese orthopedics association score (JOAS) at 3 weeks, 3 months and 6 months. There is a significant decrease between preoperative VAS and at 6 months, in both PLF and PLIF group. JOAS was significantly increased at 6 months in both PLF and PLIF group as compared to preoperative score. But difference in JOAS at 6 months is not significant between PLF and PLIF.

Conclusions: Both PLF and PLIF are equally effective for spondylolisthesis. Both techniques have same satisfactory results. As PLIF is more invasive technique, more operative time and more complications are seen.
\end{abstract}

Keywords: Spondylolisthesis, Posterior lumbar fusion, Posterior lumbar interbody fusion

\section{INTRODUCTION}

Low back pain is the most common cause of work-related disability and one of the most common contributors to missed time at work. ${ }^{1}$ Spondylolisthesis is derived from the Greek words spondyl (vertebra) and olisthesis (to slip). This most commonly describes the forward slippage of a cephalad vertebra on a caudal vertebra. ${ }^{2}$ Two processes, dysplastic and traumatic can give rise to spondylolisthesis. These can occur simultaneously, but generally one precedes the other. ${ }^{3}$ There are various treatment options available for spondylolisthesis. They are conservative, epidural steroid injections, only decompression, pars repair, PLF, PLIF, transforaminal interbody fusion and circumferential fusion. Conservative treatment for segmental instability is possible for patients with tolerable pain. ${ }^{4}$ Surgery is indicated if symptoms are disabling and interfere with work, if the condition is progressive, or if there is a significant neurological deficit. ${ }^{5}$ 
The main goals of posterior instrumentation are to give stability to the segment and to contain displacement. ${ }^{6}$ There are various studies on functional outcome of individual PLF and PLIF in patient with spondylolisthesis. These studies show good or excellent result with both these techniques. There are very less studies which compare both these techniques in cases of spondylolisthesis. So here in this study, we compare functional outcome of both these techniques.

\section{Aim}

The aim was to analyze whether the new procedure of PLIF has any advantage in terms of functional outcome over the traditional PLF.

\section{Objective}

The objective was to compare the functional outcome between PLF (intertransverse fusion) with pedicle screw fixation and PLIF (interbody cage) with pedicle screw fixation in spondylolisthesis. Assessment of the outcome was proposed to be done by specific benchmarks as given in the JOAS.

\section{METHODS}

The study was conducted in the department of orthopedics at a tertiary care centre in Rajasthan from January 2019 to July 2020.

\section{Inclusion criteria}

All patients of age $\geq 18$ years, having consented after being duly informed and having suggestive history and objective evidence, both clinical and radiological, of spondylolisthesis (all grades and types) were included in this study.

\section{Exclusion criteria}

History of failed back syndrome, neoplastic conditions of spine, associated with other pathological conditions like infection, neurological or neuromuscular disorder patients were excluded from this study.

Total 20 patients with spondylolisthesis were allotted alternatively in PLF group and PLIF group. The detailed history was taken and thorough general physical examination was done with special emphasis on features of spondylolisthesis. Systemic examination was done along with routine blood investigations, X-rays of lumbar spine (anteroposterior and lateral views) along with dynamic flexion-extension view and MRI scan of spine were done. Apart from a detailed physical examination of spine, neurological examination was also done. All the above data were documented in the study proforma.

The surgery performed was decompression and posterior instrumentation, with either PLF or PLIF.

\section{Surgical procedure}

The patient was given general anesthesia and positioned prone over two longitudinal bolsters below the trunk and abdomen free in-between the bolsters. Hip was in neutral position. Knee was in slight flexion. Vertebral levels to be fused were provisionally surface marked with the help of IITV in both AP and lateral plane. The patient was painted and draped. 15-20 $\mathrm{ml}$ of 1:1000 adrenaline diluted in 100 $\mathrm{ml}$ normal saline was infiltrated in paraspinal muscle. Straight, longitudinal and midline incision given. After proper dissection, pedicle screws were inserted at junction of the superior facet and the transverse processes with free hand targeting technique. Decompression by laminectomy was done at the compressed level, if spondylolisthesis is associated with any lumbar canal stenosis. Facetectomy or foraminotomy was done if there is any foraminal stenosis. After applying titanium rod, distraction and reduction of listhesis was done as much as possible.

In PLF after pedicle screw fixation, bone graft from laminectomy or foraminotomy was placed between transverse processes and around the facets on both the sides. In PLIF Titanium cage of measured size filled with bone graft was inserted between vertebral bodies.

Any improvement in symptoms was noted, besides neurological assessment using Frankel scale and functional assessment post operatively at 6 weeks, 3 months and 6 months. Overall clinical results were determined by the JOAS and VAS.

Data was entered in MS excel software version 10. Further it was analysed using SPSS statistical software version 21 IBM corporation. Descriptive data was presented as mean, $\mathrm{SD}$, proportions using contingency tables. Categorical data was analysed using chi square test and Mc-Newman test. Quantitative data was analysed using student t test (paired and unpaired). $\mathrm{P}$ value below 0.05 was considered significant.

\section{RESULTS}

10 patients underwent PLF and another 10 patients underwent PLIF. Mean age of patients in PLF group was 58.4 years and in PLIF group was 50.2 years. The overall mean age was 54.3 years (47 to 70 years). In our series of 20 patients, $7(35 \%)$ were males and $13(65 \%)$ were females. In PLF group, 4 (40\%) were males and 6 (60\%) were females. In PLIF group $3(30 \%)$ were males and 7 (70\%) were females. We had $1(5 \%)$ patient with L3-L4 level spondylolisthesis, 14 (70\%) patients with L4-L5 level spondylolisthesis and $5(25 \%)$ patients with L5-S1 level spondylolisthesis. Hence, L4-5 level was the commonest in both the groups.

Out of 20 patients, $4(25 \%)$ were of grade $1,14(70 \%)$ were of grade 2, 2 (10\%) were of grade 3 and none of grade 4 . In PLF group, 2 patients were of grade 1,8 of grade 2 and none of grade 3 and 4 . In PLIF group, 2 patients were of 
grade 1, 6 of grade 2, 2 of grade 3 and none of grade 4 . In our series of 20 patients, $20(100 \%)$ had back pain, 17 $(85 \%)$ had radiculopathy and $11(55 \%)$ had numbness. 20 $(100 \%)$ patients had tenderness over spine, $12(60 \%)$ patients had step, $14(70 \%)$ patients had SLR $<60,3(15 \%)$ patients had motor deficit and 5 (25\%) patients had sensory deficit. In our study, preoperatively, 17 patients had their neurological status assessed as Frankel scale E, 2 patients (1 of PLF group and 1 of PLIF group) had Frankel scale D, 1 patient (PLF group) had Frankel scale C postoperatively, all patients with a preoperative deficit improved to have a Frankel scale rating of $\mathrm{E}$ at 6 months follow up. In our study, total 5 patients (2 of PLF group and 3 of PLIF group) had sensory deficit preoperatively. Among them 3 patients show sensory recovery on their 6 months follow up. In PLF group, out of 2 patients, 1 patient showed sensory recovery, while in PLIF group, out of 3 patients, 2 patients showed sensory recovery.

Average operative time was $120 \mathrm{~min}$ in PLF group and 150 min in PLIF group. The difference in time was significant.

Table 1: Grades of spondylolisthesis.

\begin{tabular}{|lllll|}
\hline Grade & PLF & PLIF & Total & $\%$ \\
\hline Grade 1 & 2 & 2 & 4 & 25 \\
\hline Grade 2 & 8 & 6 & 14 & 70 \\
\hline Grade 3 & 0 & 2 & 2 & 10 \\
\hline Grade 4 & 0 & 0 & 0 & 0 \\
\hline
\end{tabular}

Table 2: VAS score.

\begin{tabular}{|llllll|}
\hline Group & Pre-op & 3 weeks & 3 months & 6 months & $\begin{array}{l}\text { P value (preop versus } \\
\text { 6 months) }\end{array}$ \\
\hline PLF & 5.9 & 4.1 & 3.1 & 2.1 & $<0.0001$ \\
\hline PLIF & 6.3 & 3.9 & 2.9 & 1.9 & $<0.0001$ \\
\hline P value (PLF versus PLIF) & 0.65 & 0.748 & 0.657 & 0.483 & \\
\hline
\end{tabular}

Table 3: Japanese orthopedics association.

\begin{tabular}{|llll|}
\hline JOAS & PLF & PLIF & P value (PLF versus PLIF) \\
\hline Pre-op & 15 & 14.8 & 0.904 \\
\hline 3 weeks & 19.6 & 20.4 & 0.536 \\
\hline 3 months & 22.9 & 23.8 & 0.483 \\
\hline 6 months & 24.2 & 24.9 & 0.549 \\
\hline P value (pre-op versus 6 months) & $<0.0001$ & $<0.0001$ & \\
\hline
\end{tabular}

Table 4: VAS score comparison.

\begin{tabular}{|llllll|} 
& PLF group & & PLIF group & P value (PLF \\
& Pre-op & 6 months & Pre-op & 6 months & versus PLIF) \\
\hline Our study & 5.9 & 2.1 & 6.3 & 1.9 & 0.483 \\
\hline Ching-Hsiao Yu et al & 5.6 & 2.0 & 6.0 & 1.7 & 0.355 \\
\hline Kim KT et al & 5.8 & 2.1 & 6.2 & 1.8 & 0.389 \\
\hline
\end{tabular}

Table 5: Japanese orthopedics association comparison.

\begin{tabular}{|lll|lll|}
\hline & PLF group & & PLIF group & P value (PLF \\
\cline { 2 - 6 } & Pre-op & 6 months & Pre-op & 6 months & 0.549 \\
\hline Our study & 15 & 24.8 & 14.8 & 24.9 & 0.355 \\
\hline Shugo Kuraish et al & 12.9 & 24.2 & 14.8 & 23.4 & 0.389 \\
\hline DN Inamdar et al & 13.8 & 24.6 & 14.6 & 24.1 & PLIF) \\
\hline
\end{tabular}




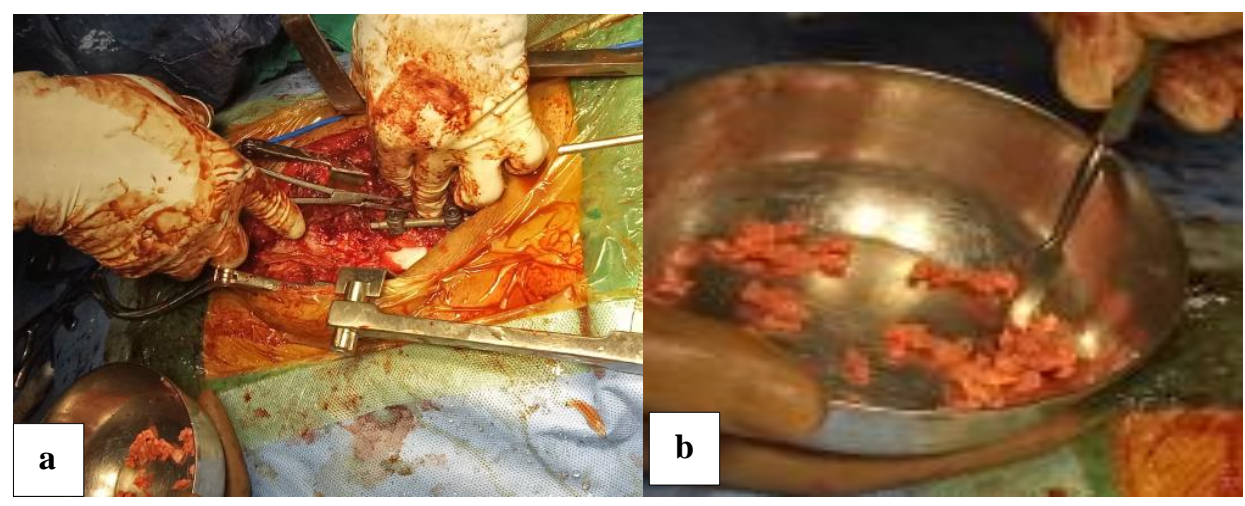

Figure 1: (a, b) intraoperative PLF.

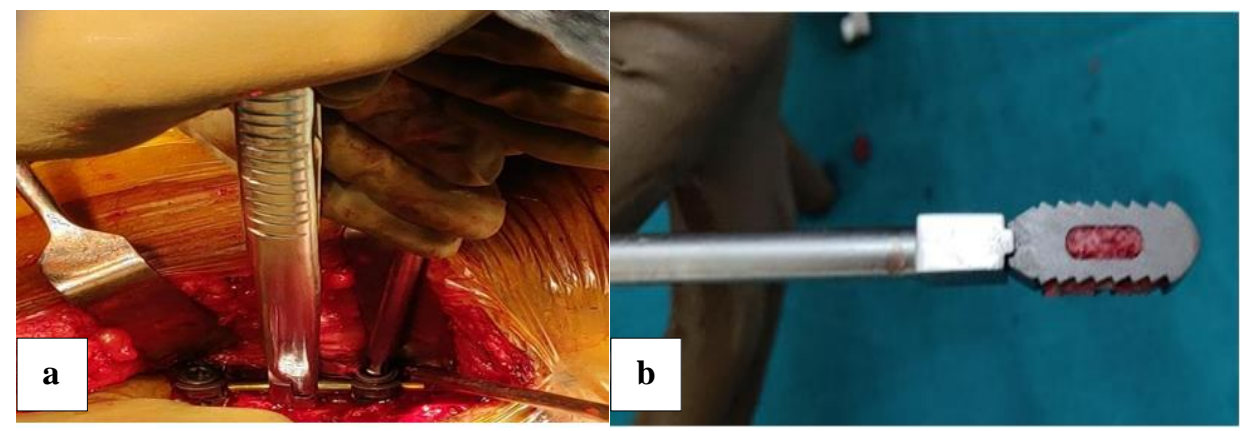

Figure 2: (a, b) intraoperative PLIF.
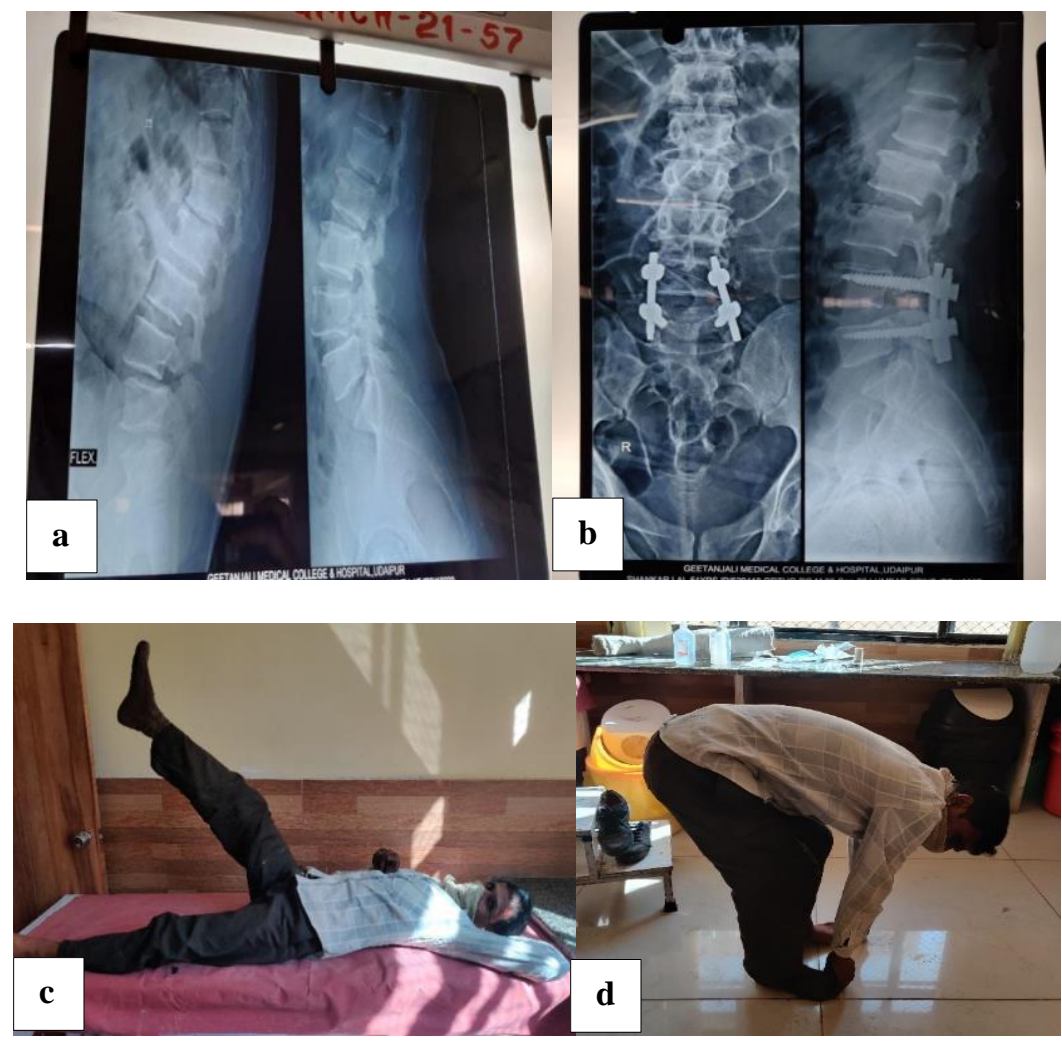

Figure 3: (a, b) Preop X-ray with grade 2 spondylolisthesis L4-L5 level; (c, d) treated with PLF; post op X-ray and movements at final follow up. 
In PLF group average VAS score at preop was 5.9, at 3 weeks was 4.1 , at 3 months was 3.1 and at 6 months was 2.1. $\mathrm{P}$ value of the difference in VAS between preop and 3 months is 0.004 , so it is statistically significant. $\mathrm{P}$ value of the difference between preop and 6 months is $<0.0001$, so it is also statistically significant. In PLIF group, average VAS score at preop was 6.3 , at 3 weeks was 3.9 , at 3 months was 2.9 and at 6 months was 1.9. $\mathrm{P}$ value of the difference in VAS between preop and 3 months is 0.005 , so it is statistically significant. $\mathrm{P}$ value between preop and 6 months is $<0.0001$, so it is statistically significant. P value between PLF and PLIF group at preop, 3 weeks, 3 months, 6 months is $>0.05$, so difference in VAS score between PLF and PLIF is not statistically significant.

In PLF group, average JOAS score at preop was 15 , at 3 weeks was 19.6 , at 3 months was 22.9 and at 6 months was 24. $\mathrm{P}$ value of the difference in JOAS between preop and 3 months is 0.004 , so it is statistically significant. $P$ value between preop and 6 months is $<0.0001$, so it is also statistically significant. In the PLIF group, average JOAS score at preop was 14.8 , at 3 weeks was 20.4 , at 3 months was 23.8 and at 6 months was 24.9. $\mathrm{P}$ value of the difference in JOAS between preop and 3 months is 0.003 , so it is statistically significant. $\mathrm{P}$ value between preop and 6 months is $<0.0001$, so it is statistically significant. $\mathrm{P}$ value of the difference in JOAS score between PLF and PLIF group at preop, 3 weeks, 3 months, 6 months is $>0.05$, so difference in JOAS score between PLF and PLIF is not statistically significant.

One patient in PLIF group had intra operative dural injury due to pedicle screw breaching the medial wall of pedicle. In that case dura was repaired intraoperatively. No other postoperative complication such as infection, screw misplacement, screw loosening or migration, neurological worsening was seen in any of the groups. Total 2 patients had persistence of LBP, radiculopathy and numbness (1 in PLF group and 1 in PLIF group) till last follow up.

\section{DISCUSSION}

Low back pain is one of the most common conditions seen in orthopedics practice. Of the various causes, spondylolisthesis is common and predominant cause. ${ }^{1}$ Spondylolisthesis occurs in about $5-7 \%$ of population. ${ }^{2}$ Various surgical and non-surgical methods of treatment for spondylolisthesis have been described. Till now the exact treatment for the spondylolisthesis is a controversy. Surgical and conservative treatment is being done for lowgrade spondylolisthesis and both have positive results, although in some cases surgery gives better relief. ${ }^{7}$ Moller reported a prospective randomized study with 111 patients with spondylolisthesis and with a follow-up of two years. Patients were submitted to conservative treatment or a posterolateral surgical approach with or without placement of pedicle screws. They concluded that surgery provided better clinical outcomes and pain improvement. ${ }^{8}$
Treating a high-grade spondylolisthesis is a difficult task because of its complex pathoanatomy. ${ }^{9}$ There is no doubt that high grade spondylolisthesis is best treated by surgical means. $^{2}$ There are many surgical options at present for treating this complex pathology and each one has its own merits and demerits. Fusion at posterolateral or at interbody level or combined and which one to choose among them is also a controversy. PLF is a relatively safe technique with less rate of complications. In PLF, after reducing listhesis, it is fixed with pedicle screw and fused by putting bone graft between transverse processes and around facets. There is no need of discectomy or laminectomy unless there is any nerve root compression. ${ }^{10}$ There are various approaches to the spine with a goal of achieving solid interbody fusion, each with their own share of success and complications. Among them PLIF appears to afford the surgeon of achieving anterior column arthrodesis and posterior transpedicular instrumentation through the same incision. ${ }^{11}$

The elderly females are more prone to spondylolisthesis due to early degenerative changes. A similar rate of female preponderance has been reported in several series. In a study by Ching-HsiaoYu et al $61.8 \%$ female patients reported while Lee et al reported $67 \%$ female patients. ${ }^{12,13}$

In our study, the most common age group was 51-60 years of age and the mean age was 54.3 years. Degenerative spondylolisthesis was the most common among all as it appears after age of 50 years. ${ }^{14}$ A similar age distribution is reported by other studies such as Fernando Luiz RD et at (52.4 years), Ching-Hsiao et al (59.1 years). ${ }^{12,15}$

In our study, spondylolisthesis was found to be most common at L4-L5 level (70\%). A similar rate of L4-L5 level preponderance had been reported in several series. In a study by Fernando Luiz et at $56.6 \%$ spondylolisthesis were reported at L4-L5 level. ${ }^{15}$ Lee et al in their study found $69.33 \%$ instability at L4-L5 level. ${ }^{13}$

Our study showed $70 \%$ patient having spondylolisthesis of grade 2. A similar rate of grade 2 preponderance had been reported by Ramesh Benguluri et al. ${ }^{16}$ They found $58.13 \%$ patients having grade 2 spondylolisthesis in their study.

The difference in duration of surgery in our study was significant $(\mathrm{p}=0.0001)$ between the groups. Inamdar et al show less operative time in PLF group. ${ }^{17}$ Kuraishi et at reported significantly more operative time in PLF group than PLIF group. ${ }^{18}$

In our study pain was decided by VAS score. PLIF group had little less pain as compared to PLF group at 3 months and 6 months follow up, however it was statistically insignificant. A similar study by Ching-HsiaoYu et al noted that VAS score at 6 months follow up was not statistically significant between these two groups. ${ }^{12}$ Kim et al had reported that there were significant differences in VAS scores between preop and post op (6 months) in both 
group but no significant difference between PLF and PLIF. ${ }^{19}$

Functional outcome was evaluated by using JOAS preoperatively and postoperatively at 3 weeks, 3 months and 6 months of follow up. In total, at final follow up, we found that $5(25 \%)$ patients had excellent result, $13(65 \%)$ patients had good result and $2(10 \%)$ patients had fair result. In PLF group, 2 (20\%) had excellent result, 7 (70\%) had good result and $1(10 \%)$ had fair result. In PLIF group $3(30 \%)$ had excellent result, $6(60 \%)$ had good result and $1(10 \%)$ had fair result. $\mathrm{P}$ value of the difference in JOAS score between the two groups was 0.549 at 6 months. PLIF group had better improvement in JOAS than PLF group. But the difference was not statistically significant. Similar results have been found in various other studies. Shugo Kuraishi et al found that clinical outcomes by JOA scores were not significantly different between the two groups. ${ }^{18}$ Post fixation spondylolisthesis correction was better in the PLIF group. However, no significant difference was observed for lumbar lordosis between the two groups. Fernando Luiz Rolemberg Dantas et at found better functional outcome with PLIF group than PLF group. ${ }^{15}$

DN Inamdar et at found that $100 \%$ patients had satisfactory (excellent or good) result in PLF group and $87 \%$ had satisfactory result in PLIF group. ${ }^{17}$ But the difference in clinical outcome was not significant. They concluded that PLF achieves a greater improvement in functional outcome than PLIF. PLIF ( $48 \%$ correction) provides better correction of spondylolisthesis than PLF (39\% correction). The fusion rates in both PLF and PLIF patients were equal $(100 \%)$.

In our study, one patient had dural tear intraoperatively in PLIF group. In that patient dura was repaired intraoperatively. There was no intraoperative complication seen in PLF group.

Among 20 patients, 2 patient had persistence of symptoms (LBP, radiculopathy and numbness) at 6 months follow up. 1 patient was of PLF group and 1 patient was of PLIF group. No patient had infection, screw loosening, screw migration or neurological worsening.

Some other study show more complication in PLIF. Shugo Kuraishi et al show higher number of complications in the PLIF group might have resulted from a more invasive nature of total facetectomy or discectomy in comparison with PLF. ${ }^{18}$

Fernando Luiz et al found that PLF patients presented with a higher complication rate such as loosening of the construct and nerve root injury. ${ }^{15}$ No such complications occurred in PLIF patients. This difference was statistically significant. The overall re-operation rate was $6.6 \%$ in PLF ( 2 cases) and $20 \%$ in PLIF (6 cases).

\section{CONCLUSION}

Both PLF and PLIF were equally effective for spondylolisthesis. Both techniques had same satisfactory results. As PLIF was more invasive technique, more operative time and more complications were seen.

Funding: No funding sources

Conflict of interest: None declared

Ethical approval: The study was approved by the institutional ethics committee

\section{REFERENCES}

1. O'Donnell JB. A biomechanical comparison of an interspinous fixation device and a pedicle screw and rod system for posterior fixation. Sem Schol. 2010.

2. Panjabi MM. Clinical spinal instability and low back pain. J Electromyograph Kinesiol. 2003;13(4):371-9.

3. Hensinger RN. Spondylolysis and spondylolisthesis in children and adolescents. JBJS. 1989;71(7):1098107.

4. Laurent LE, Einola S. Spondylolisthesis in children and adolescents. Acta Orthopedicsa Scandinavica. 1961;31(1):45-64.

5. Scot Boden D,Riew Daniel,etal. Biomechanics of spondylolisthesis. J Bone Joint Surg. 1996:403-11.

6. Möller H, Hedlund R. Surgery versus conservative management in adult isthmic spondylolisthesis: a prospective randomized study: part 1. Spine. 2000;25(13):1711-5.

7. Cloward RB The treatment of ruptured intervertebral discs by vertebral body fusion: Indications, operative technique, after care. J neurosurgery. 1953;10(2):154-68.

8. Bogduk N. Clinical anatomy of the lumbar spine and sacrum. 5th ed. London: Churchill Livingstone; 2005.

9. Arslan M, Comert A, Acar HI, Ozdemir M, Elhan A, Tekdemir I, et al. Surgical view of the lumbar arteries and their branches: an anatomical study. Op Neurosurg. 2011;68(1):16-22.

10. Wiltse LL. Spondylolisthesis: classification and etiology: symposium of the spine. Am Acad Orthop Surg. 1969:143-5.

11. Louis R. Fusion of the lumbar and sacral spine by internal fixation with screw plates. Clinical Orthopedics and Related Research. 1986;203:18-33.

12. Edeinken. Spondylolisthesis in children and adolescents. Bone Joint Surg. 1981;63(1):67-70.

13. Koslosky E, Gendelberg D. Classification in brief: the Meyerding classification system of spondylolisthesis. Assoc Bone Joint Surg. 2020;478(5):1125-30.

14. Belfi LM, Ortiz AO, Katz DS. Computed tomography evaluation of spondylolysis and spondylolisthesis in asymptomatic patients. Spine. 2006;31(24):907-10. 
15. Laasonen EM, Ehrström J. Myelography in lumbar spondylolisthesis. Acta Radiological Diagnosis. 1978;19(6):889-96.

16. Roca J, Ubierna MT, Cáceres E, Iborra M. One-stage decompression and posterolateral and interbody fusion for severe spondylolisthesis: an analysis of 14 patients. Spine. 1999;24(7):709-14.

17. Boxall D, Bradford DS, Winter RB, Moe JH. Management of severe spondylolisthesis in children and adolescents. J Bone Joint Surg. 1979;61(4):47995.

18. Kalichman L, Hunter DJ. Diagnosis and conservative management of degenerative lumbar spondylolisthesis. European Spine Journal. 2008;17(3):327-35.
19. Polly DW. Epidural steroids for degenerative spondylolisthesis: good, bad, or indifferent? Commentary on an article by Gerling MC, MD, et al.: epidural steroid injections for management of degenerative spondylolisthesis. little effect on clinical outcomes in operatively and nonoperatively treated patients". J Bone Joint Surg. 2020;102(15):1378.

Cite this article as: Singh $\mathrm{H}$, Patel D, Tyagi $\mathrm{S}$, Saoji K, Patel T, Patel P. A comparative study of functional outcome between posterior lumbar fusion and posterior lumbar interbody fusion in spondylolisthesis. Int J Res Orthop 2021;7:544-50. 